5

6

7

8

9

9

\title{
Effects of background and contour luminance on the hue and
}

\author{
brightness of the Watercolor effect
}

Peggy Gerardin ${ }^{2}$, Michel Dojat ${ }^{3}$, Kenneth Knoblauch $^{2,}$, Frédéric Devinck ${ }^{1 *}$,

${ }^{1}$ Université Rennes 2, LP3C, EA 1285, 35000 Rennes, France

${ }^{2}$ Univ Lyon, Université Claude Bernard Lyon 1, Inserm, Stem Cell and Brain Research Institute U1208, 69500 Bron, France. 3 Université Lyon 1, 69003 Lyon, France

${ }^{3}$ Univ. Grenoble Alpes, Inserm, CHU Grenoble Alpes, GIN, 38000 Grenoble, France

* Corresponding author. Tel.: +33-2-99-19-59; Fax: +33-2-99-14-19-54

e-mail address: frederic.devinck@univ-rennes2.fr

Present address: Département de Psychologie, Université Rennes 2, 35043

Rennes Cedex, France. 


\section{Abstract}

Conjoint measurement was used to investigate the joint influences of the luminance of the background and the inner contour on hue- and brightness fillingin for a stimulus configuration generating a water-color effect (WCE), i.e., a wiggly bi-chromatic contour enclosing a region with the lower luminance component on the exterior. Two stimuli with the background and inner contour luminances covarying independently were successively presented, and in separate experiments, the observer judged which member of the pair's interior regions contained a stronger hue or was brighter. Braided-contour control stimuli that generated little or no perceptual filling-in were also used to assess whether observers were judging the interior regions and not the contours themselves. Three nested models of the contributions of the background and inner contour to the judgments were fit to the data by maximum likelihood and evaluated by likelihood ratio tests. Both stimulus components contributed to both the hue and brightness of the interior region with increasing luminance of the inner contour generating an assimilative filling-in for the hue judgments but a contrast effect for the brightness judgments. Control analyses showed negligible effects for the order of the luminance of the background or inner contour on the judgments. An additive contribution of both components was rejected in favor of a saturated model in which the responses depended on the levels of both stimulus components. For the hue judgments, increased background luminance led to 
43 greater hue filling-in at higher luminances of the interior contour. For the

44 brightness judgments, the higher background luminance generated less brightness

45 filling-in at higher luminances of the interior contour. The results indicate 46 different effects of the inner contour and background on the induction of the

47 brightness and coloration percepts of the WCE, suggesting that they are mediated 48 by different mechanisms.

49

50

51 Keywords: filling-in, color assimilation, watercolor effect, color appearance, 52 scaling, conjoint measurement, MLCM. 


\section{Introduction.}

Color appearance is not determined only by the local light signals from each object but is also influenced by global contextual features. The watercolor effect (WCE) is an interesting phenomenon for studying such processes (Pinna, 1987; Pinna et al., 2001). A pair of wiggly contours composed of a light chromatic contour (e.g., orange) surrounded by a darker chromatic contour (e.g., purple) bounding an achromatic surface area elicits a filling-in of the hue of the lighter contour over the entire enclosed area (Figure 1a). The WCE is distinguished from other assimilation illusions by its large spatial extent; the phenomenon has been observed over distances of up to 45 deg (Pinna et al., 2001). In addition to the assimilative color spreading, the subjectively colored area is perceived as figure while the surrounding area appears as ground (Pinna et al., 2003; Pinna \& Tanca, 2008; Tanca \& Pinna, 2008).

\section{Figure 1 about here}

Studies of the WCE have typically examined the effects of the inducer configuration producing the WCE. For example, the intensity of the filling-in percept appears greater with increases in luminance contrast between the inner and outer contours for an achromatic WCE (Cao et al., 2011) and for a WCE that has both luminance and chromatic components (Devinck et al., 2005; Devinck \& Knoblauch, 2012). Devinck et al. (2005) noted that observers did 
not need to modify significantly the luminance of the enclosed area in a matching experiment. Other critical characteristics of the inducing contours that modulate the strength of the WCE include the continuity and contiguity of the contour pairs (Devinck \& Spillmann, 2009; Devinck \& Knoblauch, 2012). Recent demonstrations of the sensitivity of the phenomenon to contour adaptation provide additional support for a role of contour integration mechanisms in the WCE (Coia \& Crognale, 2017). The strength of the phenomenon was found to be size-tuned with the strongest WCE observed for a contour width of about 15 arcmin and was optimal for equal contour widths (Devinck et al., 2014a). While the WCE has been reported for linear contours (Pinna et al., 2001), its strength is nearly independent of the amplitude of contour undulation but increases with contour frequency up to an asymptotic level (Gerardin et al., 2014). Finally, Pinna et al. (2001) demonstrated that several different color pairs can generate the coloration effect (see also Devinck et al., 2005). Specifically, Devinck et al. (2006) demonstrated that the coloration effect is stronger when the chromatic contrast is larger. Thus, the coloration effect depends on a conjunction of chromatic and luminance contrasts but also on spatial parameters of the inner and outer contours.

The WCE is perceptually salient but has proved difficult to quantify with precision showing large variability within and across observers (Cao et al., 2011; Devinck et al., 2005; von der Heydt \& Pierson, 2006). More recently, the WCE was quantified by using paired-comparison methods that have been 
extended to estimate perceptual scales within a signal detection framework (Devinck \& Knoblauch, 2012). Two such procedures are Maximum Likelihood Difference Scaling or MLDS (Maloney \& Yang, 2003; Knoblauch \& Maloney, 2008, 2012) and Maximum Likelihood Conjoint Measurement or MLCM (Ho et al., 2008; Knoblauch \& Maloney, 2012). Difference scaling is useful for measuring perceptual strength along a single physical dimension, whereas conjoint measurement was conceived to assess the combined effects of several dimensions on appearance (Falmagne, 1985; Knoblauch \& Maloney, 2012; Krantz et al., 1971; Luce \& Tukey, 1964; Roberts, 1979). MLCM has been successfully applied to estimate perceptual scales associated with different sets of physical continua including surface material properties (Ho, Landy \& Maloney, 2008; Qi, Chantler, Siebert \& Dong, 2015; Hansmann-Roth \& Mamassian, 2017), color appearance (Gerardin et al., 2014; Rogers, Knoblauch \& Franklin, 2016) and time perception (Lisi \& Gorea, 2016). The signal detection decision model allows specifying the perceptual scales in terms of the signal detection parameter $d^{\prime}$ (Gerardin et al., 2014; Knoblauch \& Maloney, 2012).

The aim of the present study is to estimate perceptual scales for two dimensions, the luminance elevation of the inner contour and the luminance elevation of the background. While the luminance contrast between the inner and outer contours has been tested intensively in the WCE, experiments evaluating the influence of the background luminance are scarce. Indeed, the 
119 WCE has generally been demonstrated for a background of higher luminance

120 than both inner and outer contours. Although the surround (e.g., the

121 background) is known to be an important influence of color appearance

122 (Brenner \& Cornelissen, 2002; Brown \& MacLeod, 1997 ; Shevell, 1978 ;

123 Walraven, 1976), it has not been systematically explored for the coloration

124 effect in the WCE. In addition, most studies of the WCE focus solely on its

125 coloration effect. Here, we also investigate the influences of the background

126 and inner contour luminances on the perceived brightness of the interior region.

127 In summary, we employed conjoint measurement to study how both the 128 background and the inner contour luminances influence judgments of both the 129 hue and brightness in the WCE.

130

131

132

133

134

135

136

137

138

139

140

\section{General Methods}

\subsection{Observers}

Four observers participated in these experiments. Three were naïve and the fourth was one of the authors. Observers ranged in age between 26 and 40 years. All had normal color vision as tested with the Farnsworth Panel D15, and had normal or corrected-to-normal visual acuity. Experiments were performed in accordance with the principles of the Declaration of Helsinki for the protection of human subjects. 


\subsection{Apparatus}

143 Stimuli were presented on a NEC MultiSync FP2141sb color CRT monitor

144 driven by a Cambridge Research ViSaGe graphic board with a color resolution

145 of 14 bits per gun (Cambridge Research Systems, Rochester, United Kingdom).

146 The experimental software was written to generate all stimuli, control stimulus

147 presentation and collect responses in MATLAB 7.9 (MathWorks, 148 http://mathworks.com), using the CRS Toolbox extensions. The monitor was 149 calibrated using an OptiCal photometer with the calibration routines of 150 Cambridge Resarch Systems. Observer position was stabilized by a chinrest and 151 observer-to-screen distance was $80 \mathrm{~cm}$. Experiments were performed in a dark room. Both eyes were used for viewing.

\subsection{Stimuli}

155 The stimuli were constructed as Fourier descriptors (Zahn \& Roskies, 1972).

Each stimulus was defined with respect to a circle of 3.2 deg diameter whose radius, $r$, was modulated sinusoidally as a function of angle according to the equation:

$$
R(\theta)=r+A \sin (2 \pi f \theta)
$$

160 where $R$ is the stimulus radius at angle $\theta, r$ the average radius of the stimulus, $A$ the modulation and $f$ the frequency in cycles per revolution (cpr). In the present at $A=0.36$ (Figure 1b, left). 
All stimuli were composed of three colors: an orange inner contour $(\mathrm{x}, \mathrm{y}=$ $0.44,0.43$ ) with the luminance varying from $30.02 \mathrm{~cd} / \mathrm{m}^{2}$ to $62.74 \mathrm{~cd} / \mathrm{m}^{2}$ and a purple outer contour $\left(\mathrm{x}, \mathrm{y}=0.31,0.11 ; \mathrm{Y}=21.12 \mathrm{~cd} / \mathrm{m}^{2}\right)$, presented on a neutral white background $(x, y=0.29,0.32)$ with the luminance of the background (both outside and inside of the contours) varying between $35.56 \mathrm{~cd} / \mathrm{m}^{2}$ and 65.56 $169 \mathrm{~cd} / \mathrm{m}^{2}$. The contour pairs were each of width $16 \operatorname{arcmin}$, i.e., 8 arcmin for the inner and outer contours, each. 1979; Krauskopf, Williams \& Heeley, 1982; Derrington, Krauskopf \& Lennie, 1984). DKL color space is a three-dimensional opponent-modulation space based on the Smith and Pokorny (1975) cone fundamentals. The sum of L and

$175 \mathrm{M}$ cone excitations varies on one axis (luminance), while $\mathrm{M}$ cone excitation 176 subtracted from $\mathrm{L}$ cone excitation varies on the second axis ( $\mathrm{L}-\mathrm{M})$; and the sum 177 of $\mathrm{L}$ and $\mathrm{M}$ cone excitations subtracted from $\mathrm{S}$ cone excitation varies on the 178 third axis $(\mathrm{S}-(\mathrm{L}+\mathrm{M}))$. The DKL axes were scaled between -1 and 1 , where $+/-$ 1791 corresponds to the maximum contrast for each axis on the monitor. The 180 stimuli were specified with the purple and orange contours at azimuth of 320 181 and 45 deg respectively. Luminance of the independent variables is specified as 182 elevation from the equiluminant plane. The luminance elevations of the orange 183 contour in DKL color space varied from -0.6 to 0 while the luminance elevation 184 of the background ranged from -0.5 to 0 . 
of luminance background were used. All levels were crossed creating a $5 \times 5$ grid with a total of 25 stimuli. Figure 2 shows an example of the range of stimuli used, with the inner contour luminance varying across rows and the background luminance across columns. used to verify that observers responded to the filling-in appearance and not to other stimulus features.

\subsection{Procedure}

201 On each trial, two different stimuli chosen randomly from the $5 \times 5$ grid were presented in succession to the observer. Observers performed two tasks in

203 separate randomly ordered and counter-balanced sessions in which they 204 compared the interior regions of the two successively presented stimuli. In the 205 first task, observers were instructed to judge which central region evoked the 206 strongest orange hue. In the second task, observers were asked to judge which 
central region appeared brighter. An equal number of test and control stimuli

208

were interleaved in each session. With 5 levels along each of the dimensions

209

varied, there are $(25 * 24) / 2=300$ unordered pairs. Stimuli were randomly

ordered for each presentation. On each trial, a randomly chosen pair of test or

control stimuli was presented. A session consisted of the random presentation of

all 600 test and control pairs. Each task was repeated five times, yielding 1500

213

test and 1500 control trials for each observer.

214

Prior to the experiment, observers were dark-adapted for $3 \mathrm{~min}$. At the

beginning of each trial, a fixation cross was presented in the center of the screen

of duration $500 \mathrm{~ms}$. At its extinction, the first pattern was presented during 500

$\mathrm{ms}$ followed by a fixation cross for $500 \mathrm{~ms}$, and then the second pattern for 500

ms., followed by a blank screen. The observer's response initiated a $1 \mathrm{~s}$ pause

219 before the start of the next trial. An initial practice block of 10 trials preceded

220 the experiment. The experimental session started, when the observer felt at ease

221 with the task, otherwise additional practice sessions were run. A free viewing

222 procedure was used to ensure that observer's judgments were based on foveal

223 views of the stimuli.

\subsection{Model}

The data were analyzed as a decision process within the framework of a signal 
the best prediction of the set of observers' choices: an independence model, an additive model and a saturated model. Each model yields estimates of

231 perceptual scale values or internal responses that have the property that equal

232 differences in response are perceptually equal. The independence model fits the

233 observer's judgments based on only one of the component dimensions. The

234 additive model fits the judgments based on the sum of component psychological 235 responses generated by the physical dimensions. The saturated model fits the 236 observer's judgments including an interaction term that depends on the specific 237 levels of the two components in addition to their simple additive combination.

238 The three models are then evaluated using a nested likelihood ratio test. This is

239 done separately for the experiments based on hue, and brightness judgments.

240 The formal description of the model is described next and follows similar 241 descriptions elsewhere (Gerardin et al., 2014; Ho et al., 2008; Knoblauch \& 242 Maloney, 2012).

We represent the stimulus levels along the two dimensions by a variable

$244 \phi_{i, j}$, where $i$ and $j$ correspond to the luminance levels of the background and the 245 inner contour, respectively. In the decision models, each of the dimensions 246 contributes a response, $\psi_{i}^{1}, \psi_{j}^{2}$, to the intensity of the perceived filling-in 247 depending on the corresponding physical intensity levels, where the superscripts 248 correspond to the responses to the background and interior contour luminances, 249 respectively. In the additive model, when observers judge which central area is 
the more saturated orange color or which appears to be brighter, we suppose that

251

252

253

254

255

256

257

258

259

260

261

262

263

264

265

$$
\Delta(i, j, k, l)=\left(\psi_{i}^{1}+\psi_{j}^{2}\right)-\left(\psi_{k}^{1}+\psi_{l}^{2}\right)+\varepsilon
$$

where $\varepsilon$ refers to additive noise in the decision process and is modeled as a Gaussian random variable with $\mu=0$ and variance $=\sigma^{2}$. In plots, we indicate the stimulus level by the index and not by the physical units, allowing both dimensions to be plotted together. With 5 levels along each dimension, there are $2 * 5$ levels plus 1 variance $=11$ parameters to estimate. To make the model identifiable, however, the response at the lowest level along each dimension is arbitrarily set to $0, \psi_{1}^{1}=\psi_{1}^{2}=0$, and the variance is fixed to 1 for each estimated value, yielding only 8 parameters to estimate. The parameter values, $\psi_{i}^{j}$ are chosen to maximize the likelihood, $\mathcal{L}(\Psi ; R)$, over the ensemble of choices, $R$, made by the observer.

$$
\mathcal{L}(\Psi ; R)=\prod_{i} \Phi\left(\frac{\Delta_{i}}{2}\right)^{R_{i}}\left(1-\Phi\left(\frac{\Delta_{i}}{2}\right)\right)^{1-R_{i}}
$$

where $\Phi$ is a cumulative distribution function for a Gaussian with mean 0 and variance 1. The 2 in the denominator of the argument scales the variance for 
271 terms of $d^{\prime}$. In practice, this is performed using a Generalized Linear Model

272 with the MLCM package in R (Knoblauch \& Maloney, 2012, 2014).

273

If the observer's judgments depend on only one of the component

274 dimensions, we obtain the independence model, reducing the decision variable

275 to

276

$$
\Delta(i, j, k, l)=\psi_{i}^{1}-\psi_{k}^{1}+\varepsilon
$$

277 where the judgments depend on only dimension 1, here. In this reduced model, 278 the values of $\psi_{j}^{2}$ are fixed at 0 and there are only 4 free parameters to estimate.

279 Replacing the superscript 1 by 2 yields the independence model for the other 280 dimension.

Finally, the saturated observer model includes an interaction factor that 282 depend on the intensity levels of both dimensions; the decision variable is 283 defined as follows:

$$
\Delta(i, j, k, l)=\left(\psi_{i}^{1}+\psi_{j}^{2}+\psi_{i j}^{12}\right)-\left(\psi_{k}^{1}+\psi_{l}^{2}+\psi_{k l}^{12}\right)+\varepsilon
$$

285 Due to the interaction terms, the responses cannot be explained by a simple 286 additive combination of components as in the previous two models. With 5 287 levels along each dimension, only one cell in the $5 \times 5$ grid of responses is fixed 288 at 0 leading to 24 (the maximum) free parameters to estimate, which is the 289 origin of the term saturated. 
perceptual scale values and model the contribution of both dimensions. The

293 likelihood ratio tests were evaluated using a $\chi^{2}$ statistic with degrees of freedom

294 the difference in number of parameters fit for each pair of models.

\section{Results}

297 Judgments based on color and on brightness from the observers are shown in 298 Figure 3 for test and control conditions in Conjoint Proportion Plots or CPP (Ho 299 et al., 2008; Knoblauch \& Maloney, 2012). In the CPP, the raw data are 300 presented in a grid format in which each cell of the grid corresponds to one 301 stimulus pair comparison. Each CPP contains all stimulus combinations and 302 summarizes the proportion of times the stimulus $S_{k l}$ was judged for one response

303 criterion, hue (a) or brightness (b), to show a greater filling-in than the stimulus $304 S_{i j}$, coded according to the grey levels indicated by the color bar presented on the 305 right side of each graph. The levels of both dimensions are represented along 306 each axis where the $5 \times 5$ outer check indicates the stimulus levels along one 307 dimension and with each outer check subdivided into smaller $5 \times 5$ checks 308 indicating the stimulus levels for the second dimension. Figure $3 \mathrm{c}$ shows the 309 expected pattern of responses for an ideal observer who chooses only the higher 310 level along one of the two stimulus dimension. The CPP presented on the left 311 side indicates the results when the judgments depend on the first dimension 312 alone (here, the background luminance) and the CPP displayed on the right side 
313 when the judgment depend on the second dimension (here, the inner contour 314 luminance).

Results from the hue and brightness judgments for each observer are

316 shown in Figure $3 \mathrm{a}$ and $3 \mathrm{~b}$ respectively with the results displayed on the top row

317 for the test condition and on the bottom row for the control condition in each

318 figure. For the hue judgments, the CPP for the test stimuli resembles more

319 closely the ideal CPP displayed for the second dimension, suggesting that the

320 luminance of the inner contour contributed more strongly to the choices than the

321 luminance of the background. For the brightness judgments, the CPP for the test

322 stimuli is more similar to the ideal CPP displayed for the first dimension,

323 indicating that the luminance of the background contributed more strongly to the

324 choices in comparison with the luminance of the inner contour. Deviations from

325 the ideal patterns, however, indicate contributions from both dimensions for 326 both tasks.

In these experiments, observers were instructed to judge the appearance of 328 the interior region of the stimulus. However, it is possible that observers 329 attended to the experimental dimensions (e.g., the continuity of the color of the 330 contour) instead of the appearance of the interior region. If this were the case, 331 we should obtain the same pattern of responses between the test and the control patterns showing little, if any, systematic structure. 


\section{Figure 3 about here}

nested likelihood ratio test are shown in Table I for judgments based on hue and in Table II for judgments based on brightness. In these tables, each row indicates the test for an observer, and the last column corresponds to the probability that the additive model fits no better than the independence model.

342 The degrees of freedom are obtained from the difference of the number of 343 coefficients estimates in each model $(8$ (additive) -4 (independence) $=4)$. 344 Comparing the independence with the additive model indicates that the 345 independence model can be rejected for the test stimuli for both tasks and for all 346 observers $(p<0.001)$. The motivation for testing the nested decision models for 347 the control stimuli is less clear. Instead, we used a linear mixed-effects model to 348 test if the estimated perceptual scale values depended significantly on the 349 stimulus level with a random observer intercept (Bates et al., 2015). For both 350 the hue and the brightness judgments, no significant dependence was found 351 (hue: $\chi^{2}(30)=38.5, p=0.14$; brightness: $\chi^{2}(30)=22.2, p=0.85$ ). It could 352 be argued that 4 observers is not sufficient to estimate the variances accurately 353 in a mixed-effect model. We also performed the tests using a linear model with 354 Observer entering as a fixed-effect, with no significance obtained in either case. 355 The advantage of the mixed-effects model is that the results generalize to the 
population rather than just the sample of 4 observers tested (Knoblauch \&

Maloney, 2012; Moscatelli et al., 2013; Pinheiro \& Bates, 2000). shows the scale values estimated for the test stimulus and the bottom for the control stimulus. Black circles indicate the inner contour contribution and white the background contribution to the judgments. The values on the abscissa indicate the five stimulus levels for each dimension coded from 1 to 5 . These 370 values are indices to the 5 luminance elevations of the inner contour and the 371 background used in the experiment.

373 the interior contour dimension to the hue filling-in strength of the inner contour 374 as does the contribution of the background increases with luminance elevation 375 but with a smaller effect. The second row shows results obtained for the control 376 stimuli. Here, there appears no systematic influence of either dimension on the 377 strength of the coloration effect, because both dimension contributions are close 
to zero at all stimulus levels. This result further supports that the observers based their judgments on the perceived filled in color of the interior rather than the luminance of the inner contour or the background.

\section{Figure 4 about here}

Figure 4 (b) shows the average estimated scales for each observer for each

pairing of inner contour and background luminance elevations when judgments

were based on the brightness of the interior region. The information in the

figure is organized in the same fashion as Figure 4a. The top row shows the

additive model fits to judgments of the brightness of the interior region. As the inner contour increases in luminance, the estimated contribution for this component decreases, indicating that the brightness of the interior region decreases, i.e., generating a relative contrast rather than an assimilative effect.

The background dimension contributes positively with the luminance elevation on the strength of brightness filling-in.

397 (Yeshurun et al., 2008). To test for this, we compared the scales obtained for 398 trials in which the higher luminance was presented in the first interval with the 399 trials in which it was presented in the second interval. We performed the same 
400

401

402

403 displayed in Figure 5 (b). In these figures, the column labels indicate the

404 observer identification. The top row shows the estimated scale values for the

405 contribution of the inner contour luminance and the bottom for the background

406 dimension. White circles indicate the dimension contribution to the judgments

407 when the luminance elevation of the background is higher in the first stimulus

408 presentation and black circles the contribution to the judgments when the

409 luminance elevation of the background is higher in the second presentation. The

410 values on the abscissa indicate the five levels of each dimension coded from 1 to

4115 . For judgments based on hue, the results in Figure 5a indicate that the

412 contribution of the inner contour dimension is not different between both

413 background presentation orders. Moreover, the background dimension is

414 approximately the same between the presentation orders. Similar results were

415 obtained when judgments were based on brightness (Figure 5b). This shows

416 that any order effect based on the luminance background is very slight or absent

417 in our experiments.

For completeness, we show in Figure 6 the effect of the presentation order

419 of the luminance of the inner contour on the estimated scales for both judgments

420 organized in the same fashion as Figure 5. For both tasks, the scales for neither

421 dimension were influenced as a function of the presentation order. 

then the additive model, thus, demonstrating that an interaction term is required 428 to describe the observers' judgments for both tasks (Tables III and IV). The 429 degrees of freedom indicate the difference of the number of coefficents estimated in the 2 models $(24$ (saturated) -8 (additive) $=16)$. The additive 431 model fit was rejected in all 8 tests. The estimated coefficients for the saturated 432 model are shown for each observer and both tasks in the panels of Figure 7. In 433 these displays, the estimated scale values are plotted as a function of the 434 stimulus index for the luminance of the inner contour with the index of the 435 background luminance specified as a parameter for each curve. The results for 436 the hue judgments are presented on the top row with the brightness judgments 437 on the bottom. The averages of the four observers for both judgment conditions 438 are summarized as three-dimensional surfaces in Figures $8 \mathrm{a}$ and $\mathrm{b}$. If the 439 additive model was a good fit to the data, the curves for different background 440 levels would be parallel in Figure 7. However, for both the hue and brightness 441 responses, as the background luminance increases, the curves fan out. For the 442 hue judgments, this results in a larger range of hue responses to the range of 443 inner contour luminances tested for the higher than lower luminance 
444 backgrounds. For the brightness judgments, in contrast, the range of response 445 decreases at the highest luminance background. Thus, the background 446 luminance produces different effects on both the type of filling-in (assimilation 447 vs contrast) for hue and brightness judgments and on the dynamic range of the 448 response, expanding it for hue judgments but compressing it for brightness.

\section{Discussion}

455 In the present study, MLCM was used to quantify the contributions to the 456 filling-in strength of the WCE of two stimulus dimensions: the background and 457 inner contour luminances. We quantified how changes in these features affected 458 perceived filling-in using two separate response criteria, linked to the strength of 459 the hue (the conventional WCE) and the brightness of the interior region. 460 Control experiments using a stimulus contour that generated statistically 461 undetectable filling-in confirmed that observers judged the perceived attributes 462 of the interior regions and not changes in the background and contour 463 luminances, per se. We also found that the results were largely independent of 464 the ordering of presentation in a trial of both the backgrounds and the inner contours. 
466 As found previously, the strength of the coloration effect depends on the

467 luminance of the inner contour (Devinck \& Knoblauch, 2012; Devinck et al., 468 2014; Gerardin et al., 2014). Here, we show that the stimulus configuration 469 inducing the WCE generates both a hue and also a brightness filling-in of the 470 interior area and that these two phenomena are differentially affected by the 471 stimulus dimensions that we manipulated. The hue filling-in effect was 472 assimilative, and the hue became more saturated with increases in luminance of 473 the inner contour and the background. However, luminance of the interior 474 contour generated a contrast effect for brightness, in that the judged brightness 475 of the interior region decreased at higher contour luminances. As observers 476 compared the interior regions of the two successively displayed stimuli and 477 judged which central region appeared brighter, it is still possible that the 478 perceived filling-in was assimilative, i.e., the same contrast polarity as the inner 479 contour and that the effect of the contour luminance was simply to reduce the 480 lightness. This is difficult to assess since we cannot simply compare the interior 481 with the surround because both the surround and the interior vary in each 482 condition. Additionally, if we hold the surround constant, then we cannot rule 483 out its effect on the interior.

\section{The hue effect}

Both the hue and the brightness of the interior region were judged to be 486 greater with increases in the luminance elevation of the background. Pinna et al. 487 (2001) have previously reported that color spreading in the WCE occurs not 
488 only with a white background but also with grey backgrounds and that even a

489 faint spreading is perceived with dark backgrounds. Our results agree with these

490 observations in that the background contribution to the hue judgments increased 491 at higher luminances.

492 We also found that hue filling-in was more pronounced for bright than 493 dim backgrounds. Previous studies have reported that color saturation 494 diminishes when the brightness contrast between a colored object and its 495 luminance background increases, a phenomenon named the gamut expansion 496 effect (Brown \& McLeod, 1997) subsequently confirmed and quantified by 497 several investigators (Bimler et al., 2009; Faul et al., 2008; Xing et al., 2015). 498 In contrast with these studies, our experiment shows that increasing the 499 background luminance strengthens the assimilation hue, suggesting that we are 500 observing a different phenomenon.

\section{The brightness effect}

502 The WCE has typically been described as a coloration effect. Brightness 503 variations in the interior region of the stimulus have not typically been 504 systematically quantified. One exception to this concerns studies that used an 505 achromatic stimulus configuration (Cao et al., 2011; Coia \& Crognale, 2017). 506 In Coia and Crognale (2017), observers compared the filling-in region in the 507 WCE to a reference stimulus with a physical luminance difference. Their 508 matching results indicated that the test field shifted in the opposite direction 
509 from the inner contour showing an assimilation effect. In Cao et al. (2011), the

510 luminances of the inner contour and the luminance of the background were fixed

511 while the outer contour varied between high and low luminance levels, thus

512 varying the contrast. Observers were asked to report which of two interior

513 surface stimuli appeared darker. Their results followed a U-shape with the

514 strength of the effect maximized for a range of medium luminance levels but not

515 for the extreme luminance levels. Consequently, the luminance contrast

516 between both contours affects the WCE but not linearly. An intriguing point is

517 the authors' description of their results: "Although there is an apparent

518 assimilation effect in the chromatic WCE, it is hard to tell whether it is actually

519 assimilation or some type of contrast effect happening here for the 'opposite

520 polarity' condition". As their method based the estimation of the WCE strength

521 on the probability of discriminating which stimulus appeared darker, we can

522 assume that if responses are inferior to $50 \%$, then the surface appears lighter but

523 not equal (which would be the case for responses equal to $50 \%$ ), indicating a

524 contrast rather than an assimilation effect. For our experiment based on

525 brightness judgment, when the inner contour increases in luminance, the

526 estimated contribution for this component decreases, indicating that the

527 brightness of the interior region decreases, also indicating a contrast

528 phenomenon. Thus, the two sets of results may be in agreement. Nevertheless,

529 the nature of the brightness effect could be influenced by the presence of the

530 chromatic component in our stimulus situation. 
532 luminance and the chromaticity of a field in order to match the color of the

533 WCE. The mean luminance match was near the luminance of the background, 534 indicating that little or no luminance adjustment was required to make a 535 perceptual match. The authors concluded that the WCE is predominantly a 536 chromatic effect as originally suggested by Pinna et al. (2001). These results are not necessarily in conflict with the current study, in that we observed that the variation in brightness contrast with inner contour luminance is diminished at 539 high backgrounds and might have been difficult to detect via matching. We

540 would predict, then, that manipulating the luminance of the background would 541 affect the luminance match, but this would require a more systematic study of 542 the background than was performed by Devinck et al. (2005). Here, our data 543 indicate that the perceptual effect is not limited only to a coloration phenomenon 544 in that both the background and inner contour luminances influence observers' 545 judgment of the brightness of the central surface. A simple hypothesis to 546 account for the reduced brightness effect at high background luminances is to 547 suppose that the background light added to the interior region cancels the 548 contrast effect. This would not explain, however, the fact that higher 549 background luminances led to a stronger hue percept. 
553

554

555

556

557

558

559

560

561

562

563

564

565 571 or the other for the hue and brightness judgments.

572 573 successively displayed stimuli and judged which central region appeared 574 brighter. Under this condition, our results indicated that the brightness of the

\section{Assimilation vs contrast}

Whether one observes a contrast or assimilation effect may depend on the width of the contours (Fach \& Sharpe 1986; Helson \& Rohles, 1959; Helson, 1963). Other factors such as the luminance of the inducing stimuli can also influence our percept. Thus, de Weert and Spillmann (1995) indicated that assimilation or contrast occurred depending on whether the inducing contours of varied reflectance were darker or lighter than the gray background in using a pincushion pattern. A matching experiment for brightness judgements indicated that contrast occurred when the luminance level of the inducing contour was above the luminance level of the background and that assimilation occurred when the luminance of the inducing contour was below the luminance of the background. Given the different spatial dependencies of chromatic and luminance sensitive mechanisms, one might expect differences in the spatial domains over which chromatic and luminance components of a stimulus induce assimilation and contrast. Given the dependence of induction phenomena on stimulus configuration, however, (Fach \& Sharpe 1986; de Weert \& Spillmann 1995; Smith et al. 2001; Monnier \& Shevell 2003, 2004), it is difficult to predict a priori whether the dimensions of the contours that we used should predict one

In our study, observers compared the interior regions of the two 
575 interior region decreases generating a relative contrast rather an assimilation

576 effect. However, it still possible that different visual phenomena are perceived

577 in other circumstances. In making a brightness judgements of the central region

578 with respect to the outer region, we noted an assimilation phenomenon at the

579 lowest luminance level of the background and at the highest luminance level of

580 the inner contour. This condition corresponds to the lower left corner in Figure

5812.

582 Unitary vs multiple mechanisms

583 The color of the central surface in the WCE is characterized by a spread

584 of color from the inner contour. Most previous studies of the WCE reported that

585 the coloration effect depends on both the chromatic and luminance contrasts of

586 the inner and outer contours. For example, most authors demonstrated that the

587 coloration effect increases with increasing luminance contrast between inner and

588 outer contours (Devinck \& Knoblauch, 2012; Devinck et al., 2005).

589 Additionally, the coloration effect increases when the chromatic coordinates of

590 the inner and outer contours are approximately complementary in the color

591 diagram (Devinck et al., 2006). Thus, the main explanation assumes a filling-in

592 process in which a neuronal mechanism detects the contour and generalizes it

593 beyond the confines of the immediate stimulus. Most studies in the WCE have

594 reported an important role for several types of contour mechanisms generating a

595 long-range filling-in percept. Taken together, these data suggest that the filling-

596 in process involved in the WCE requires multiple levels of processing (Devinck 

et al., 2014b; Pinna et al., 2001; Pinna \& Grossberg, 2005; von der Heydt \& 598 Pierson, 2006). The present results indicate that the mechanisms inducing the 599 brightness and coloration percept in the WCE are affected differently by the 600 luminance of the inner contour. These opposing responses due to the inner 601 contour suggest that multiple mechanisms contribute to the appearance of the 602 interior region. Different mechanisms could be activated or inhibited yielding to 603 color assimilation or brightness contrast effects, respectively. Future 604 experiments based on visual masking or contour adaptation could investigate 605 such phenomena.

606

607

\section{Acknowledgements}

609

610 This work was supported by a grant from the Agence Nationale de la Recherche 
613

614

615

616

617

618

619

620

621

622

623

624

625

626

627

628

629

630

631

632

633

634

635

636

637

\section{References}

Bates, D., Maechler, M., Bolker, B., \& Walker, S. (2015). Fitting Linear Mixed-Effects Models Using lme4. Journal of Statistical Software, 67(1), 1-48. doi:10.18637/jss.v067.i01.

Bimler, D.L., Paramei, G.V., \& Izmailov, C.A. (2009) Hue and saturation shifts from spatially induced blackness. Journal of the Optical Society of America A, 26, 163-172.

Brown, R.O., \& MacLeod, D.I.A. (1997). Color appearance depends on the variance of surround colors. Current Biology, 7, 844-849.

Brenner, E., \& Cornelissen, F.W. (2002). The influence of chromatic and achromatic variability on chromatic induction and perceived colour. Perception, 31, 225-232.

Cao, B., Yazdanbakhsh, A., \& Mingolla, E. (2011). The effect of contrast intensity and polarity in the achromatic watercolor effect. Journal of Vision, 11(3):18, 1-8.

Coia, A.J., \& Crognale, M.A. (2017). Contour adaptation reduces the spreading of edge induced colors. Vision Research,

Derrington, A.M., \& Krauskopf, J., \& Lennie, P. (1984). Chromatic mechanisms in lateral geniculate nucleus of macaque. The Journal of Physiology, 357, 241-265.

Devinck, F., and Knoblauch, K. (2012). A Common Signal Detection Model for the Perception and Discrimination of the Watercolor Effect. Journal of Vision, 12, 1-14.

Devinck, F., and Spillmann, L. (2009). The watercolor effect: Spacing constraints. Vision Research, 49, 2911-2917.

Devinck, F., Gerardin, P., Dojat, M., and Knoblauch, K. (2014a). Spatial selectivity of the Watercolor Effect. Journal of the Optical Society of America A, 31, A1-A6.

Devinck, F., Gerardin, P., Dojat, M., and Knoblauch, K. (2014b). Quantifying the Watercolor effect: from stimulus properties to neural models. Frontiers in Human Neurosciences, 8:805. 
638 Devinck, F., Delahunt, P.B., Hardy, J.L., Spillmann, L. and Werner, J.S. (2005). The Watercolor effect: Quantitative evidence for luminance-dependent mechanisms of long-range color assimilation. Vision Research, 45, 1413-1424.

641 Devinck, F., Hardy, J.L., Delahunt, P.B., Spillmann, L., \& Werner, J.S. (2006). Illusory spreading of Watercolor. Journal of Vision, 6, 625-633.

643 DeWeert, C.M.M., \& Spillmann, L. (1995). Assimilation: asymmetry between brightness and darkness? Vision Research, 35, 1413 - 1419.

645 Fach, C., \& Sharpe, L. (1986). Assimilative hue shifts in color gratings depend on bar width. Perception \& Psychophysics, 40, 412 - 418.

647 Falmagne, J.-C. (1985). Elements of psychophysical theory. Oxford: Oxford University Press.

648 Faul, F., Ekroll, V., \& Wendt, G. (2008) Color appearance: the limited role of chromatic surround variance in the "gamut expansion effect." Journal of Vision, 8(3):30, 1-20.

Hansmann-Roth, S., \& Mamassian, P. (2017). A glossy simultaneous contrast: Conjoint measurement of gloss and lightness. i-Perception, 1-16.

Helson, H. (1960) Studies of anomalous contrast and assimilation. Journal of the Optical Society of America, 53, 179-184.

Helson H. \& Rohles F. (1959) A quantitative study of reversal of classical lightness-contrast? The American Journal of Psychology, 72, 530-538.

Ho, Y. X., Landy, M. S., \& Maloney, L. T. (2008). Conjoint measurement of gloss and surface texture. Psychological Science, 19, 196-204.

Kirschmann, A. (1890). Uber die quantitativen verhältnisse des simultanen helligkeits- und farben-contrastes. Philosophische Studien, 6, 417-491.

Knoblauch, K., \& Maloney, L. T. (2008). MLDS: Maximum likelihood difference scaling in

Knoblauch, K., \& Maloney, L. T. (2012). Modeling psychophysical data in R. New York: 
664 Knoblauch, K., \& Maloney, L. T. (2014). MLCM: Maximum Likelihood Conjoint 665 Measurement. R package version 0.4.1. https://CRAN.R-project.org/package=MLCM

Krantz, D. H., Luce, R. D., Suppes, P., \& Tversky, A. (1971). Foundations of measurement (vol. 1): Additive and polynomial representations. New York: Academic Press.

Krauskopf, J., Williams, D.R., \& Heeley, D.W. (1982). Cardinal directions of color space. Vision Research, 22, 1123-1131.

670 Lisi, M., \& Gorea, A. (2016). Time constancy in human perception. Journal of Vision, 16(4):3, 1-12.

Luce, R. D., \& Tukey, J. W. (1964). Simultaneous conjoint measurement: A new scale type of fundamental measurement. Journal of Mathematical Psychology, 32, 466-473.

MacLeod, D.I., \& Boynton, R.M. (1979). Chromaticity diagram showing cone excitation by stimuli of equal luminance. Journal of the Optical Society of America, 69, 1183-1186. Vision, 3, 573-585.

Monnier, P., \& Shevell, S.K. (2003). Large shifts in color appearance from patterned chromatic backgrounds. Nature Neuroscience, 6, 801 - 802.

Monnier, P., \& Shevell, S.K. (2004). Chromatic induction from S-cone patterns. Vision Research, 44, 849 - 856.

Moscatelli, A., Mezzetti, M., \& Lacquaniti, F. (2012). Modeling psychophysical data at the population-level: The generalized linear mixed model. Journal of Vision, 12(11):26.

Pinheiro, J.C., \& Bates, D.M. (2000). Mixed-Effects Models in S and S-PLUS. Springer, New York. Societa` Italiana di Psicologia. 
689 Pinna, B., \& Tanca, M. (2008). Perceptual organization reconsidered in the light of the

690

691

692

693

694

695

696

697

698

699

700

701

702

703

704

705

706

707

708

709

710

711

712

713 watercolor illusion: The problem of perception of holes and the object-hole effect. Journal of Vision, 8, 1-15.

Pinna, B., Brelstaff, G. \& Spillmann, L. (2001). Surface color from boundaries: a new 'watercolor' illusion. Vision Research, 41, 2669-2676.

Pinna, B., Werner, J.S. \& Spillmann, L. (2003). The watercolor effect: a new principle of grouping and figure-ground organization. Vision Research, 43, 43-52.

Qi, L., Chantler, M.J., Siebert, J.P., \& Dong, J. (2015). The joint effect of mesoscale and microscale roughness on perceived gloss. Vision Research, 115, Pt B:209-217.

R Core Team (2017). R: A language and environment for statistical computing. R Foundation for Statistical Computing, Vienna, Austria. URL https://www.R-project.org/.

Roberts, F. S. (1985). Measurement theory. Cambridge, UK: Cambridge University Press.

Rogers, M., Knoblauch, K., \& Franklin, A. (2016). Maximum likelihood conjoint measurement of lightness and chroma. Journal of the Optical Society of America A, 33, A184-A193.

Shevell, S. (1978). The dual role of chromatic background in color perception. Vision Research, 18, 1649-1661.

Smith, V.C., Jin, P.Q., \& Pokorny, J. (2001). The role of spatial frequency in color induction. Vision Research, 41, 1007 - 1021.

Tanca, M., \& Pinna, B. (2008). The phenomenal dissociation between coloration and objecthole effects in the watercolor illusion. Visual Neuroscience, 25, 423-432.

von der Heydt, R., \& Pierson, R. (2006). Dissociation of color and figure-ground effects in the watercolor illusion. Spatial Vision, 19, 323-340.

Walraven, J. (1976). Discounting the background - the missing link in the explanation of chromatic induction. Vision Research, 16, 289-295. 
714 Wiebel, C.B., Aguilar, G., \& Maertens, M. (2017). Maximum Likelihood Difference Scales

715

716

717

718

719

720 represent perceptual magnitudes and predict appearance matches. Journal of Vision, 17(4):1, 1-14.

Xing, D., Ouni, A., Chen, S., Sahmoud, H., Gordon, J., \& Shapley, R. (2015). Brightnesscolor interactions in human early visual cortex. The Journal of Neuroscience, 35, $2226-2232$.

Yeshurun, Y., Carrasco, M., \& Maloney, L.T. (2008). Bias and sensitivity in two-interval forced choice procedures: Tests of the difference model. Vision Research, 48, 18371851.

Zahn, C. T., \& Roskies, R. Z. (1972). Fourier descriptors for plane close curves. IEEE Transactions on Computers, C-21, 269-281. 
732

733

\section{Figure Legends}

Fig. 1: (a) Example of the Watercolor Effect. When a light orange contour is surrounded by a dark purple contour, the enclosed area takes the tint of the orange border. (b) Example of stimuli using Fourier descriptor as test stimulus (presented on the left side) and using braided contour as control stimulus (displayed on the right side).

Fig. 2: Examples of stimulus set used for a conjoint measurement experiment. The figure indicates the set of stimuli used in both judgment tasks. Each column corresponds to a different luminance elevation of the background and each row to a different luminance elevation of the inner orange contour.

Fig. 3: Conjoint proportion plots for judgments based on hue (a) and on brightness (b). Each plot shows the proportion of stimulus $S_{k l}$ judged to have a greater filling-in than the stimulus represented in abscissa $S_{i j}$ as grey level according to the color bar on the right side. The luminance elevation of the background is indicated by the large grids $(i, k)$ and each grid is subdivided into smaller $5 \times 5$ grid indicating the luminance elevation of the inner contour $(j, l)$. In each set of graphs, the top row indicates the results for the test stimuli and the bottom for the control 
stimuli for 4 observers. (c) Conjoint proportion plots for a simulated observer. Expected responses for an observer who judges the stimuli based on the contributions along only one of the dimensions.

757 Fig. 4: (a) Estimated scales for judgments based on hue. Additive model average estimates for test (top row) and control stimuli (bottom row) as a function of inner contour elevation (black circles) and luminance background elevation (white circles) for four observers. Error bars show 95\% confidence intervals for estimates across the 5 runs. (b) Results for judgments based on brightness. The solid lines indicate the estimated contributions of each dimension under the additive model for test (top row) and control patterns (bottom row) as a function of inner contour elevation (black circles) and luminance background elevation (white circles) for four observers. Error bars show 95\% confidence intervals for estimates across the 5 runs.

Fig. 5: (a) Results for judgments based on hue depending on the presentation order for the luminance background of the test stimulus. The solid lines indicate the estimated contributions under the additive model for the inner contour dimension (top row) and the background dimension (bottom row) as a function of the background order presentation. The white circles are used to indicate that the luminance elevation of the 
background is higher in the first interval than in the second interval and the black circles are used to represent a higher luminance elevation of the background in the second interval than in the first interval. Error bars are $95 \%$ confidence intervals based on a bootstrap procedure (Knoblauch \& Maloney, 2012). (b) Results for judgments based on brightness depending of the luminance background presentation order for the test stimulus. Additive model average estimates for the inner contour dimension (top row) and the background dimension (bottom row) when the luminance elevation of the background is higher in the first interval than in the second interval (white circles) and when the luminance elevation of the background is higher in the second interval than in the first interval (black circles). Error bars are 95\% confidence intervals based on a bootstrap procedure (Knoblauch \& Maloney,

Fig. 6: Results for judgment based on hue (a) and on brightness (b) depending on the presentation order for the luminance contour of the test stimulus. The information in the figure is organized in the same fashion as Figure 5. The white circles are used to indicate that the luminance elevation of the inner contour is higher in the first interval than in the second interval and the black circles are used to represent a higher luminance 
elevation of the inner contour in the second interval than in the first interval. Error bars are $95 \%$ confidence intervals.

798

799 Fig. 7: Results of the estimated contributions for each combination of the two dimensions under the saturated model for four observers. The different lines are used to code the index of the background dimension (for indices 1 to 5). The top row represents the estimated contribution when judgment is based on hue and the bottom row is the estimated contribution when judgment is based on brightness.

805

806 Fig. 8 Three-dimensional surfaces for the average of the data over observers from Figure 7, showing the contributions of the inner contour and background to a) the hue and b) the brightness judgments under the saturated model. 
(a)

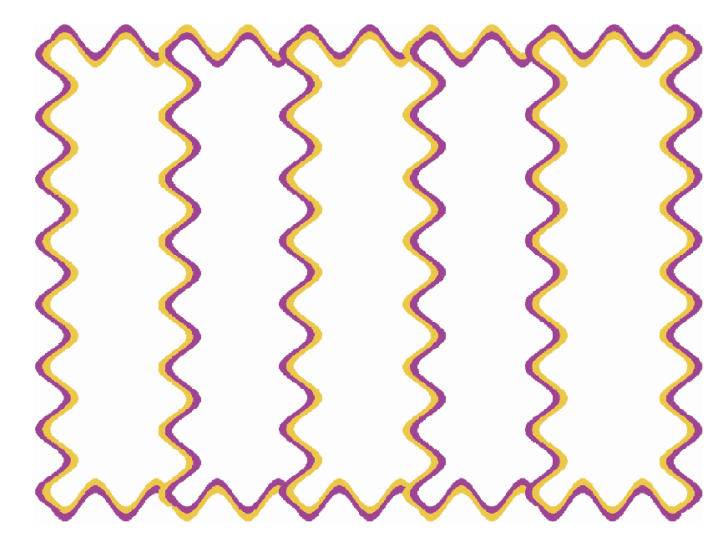

(b)
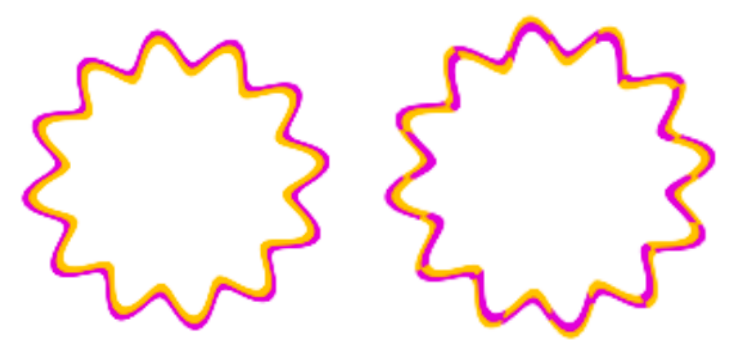

Figure 1 


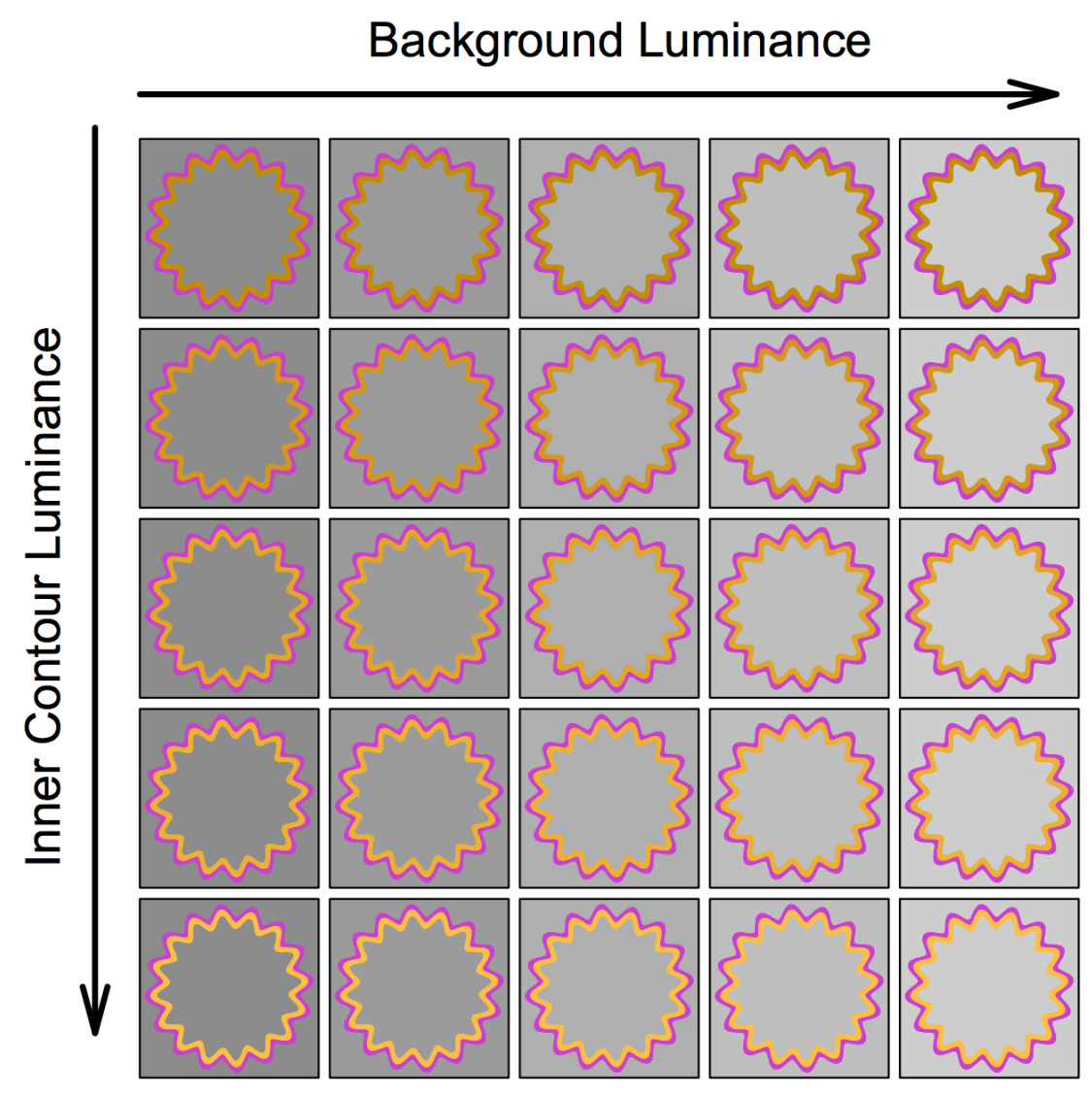

Figure 2 
(a)

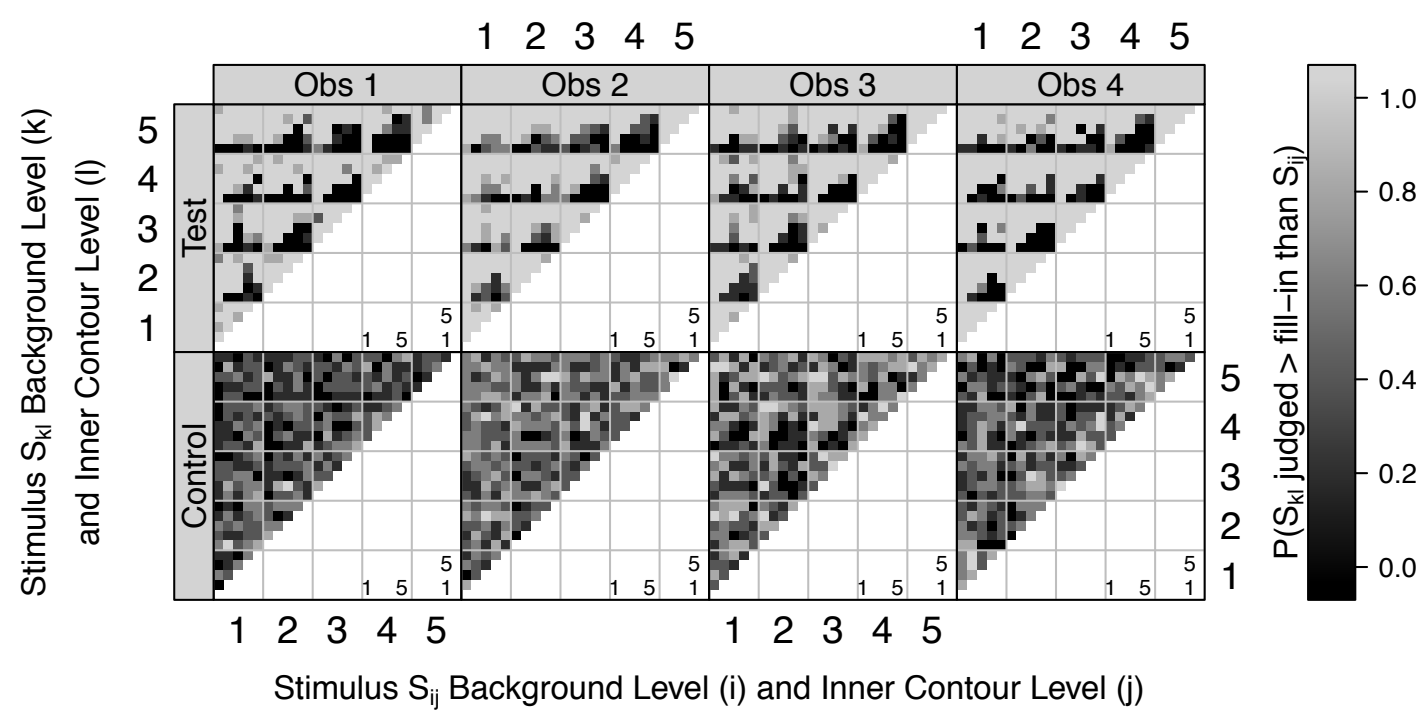

(b)

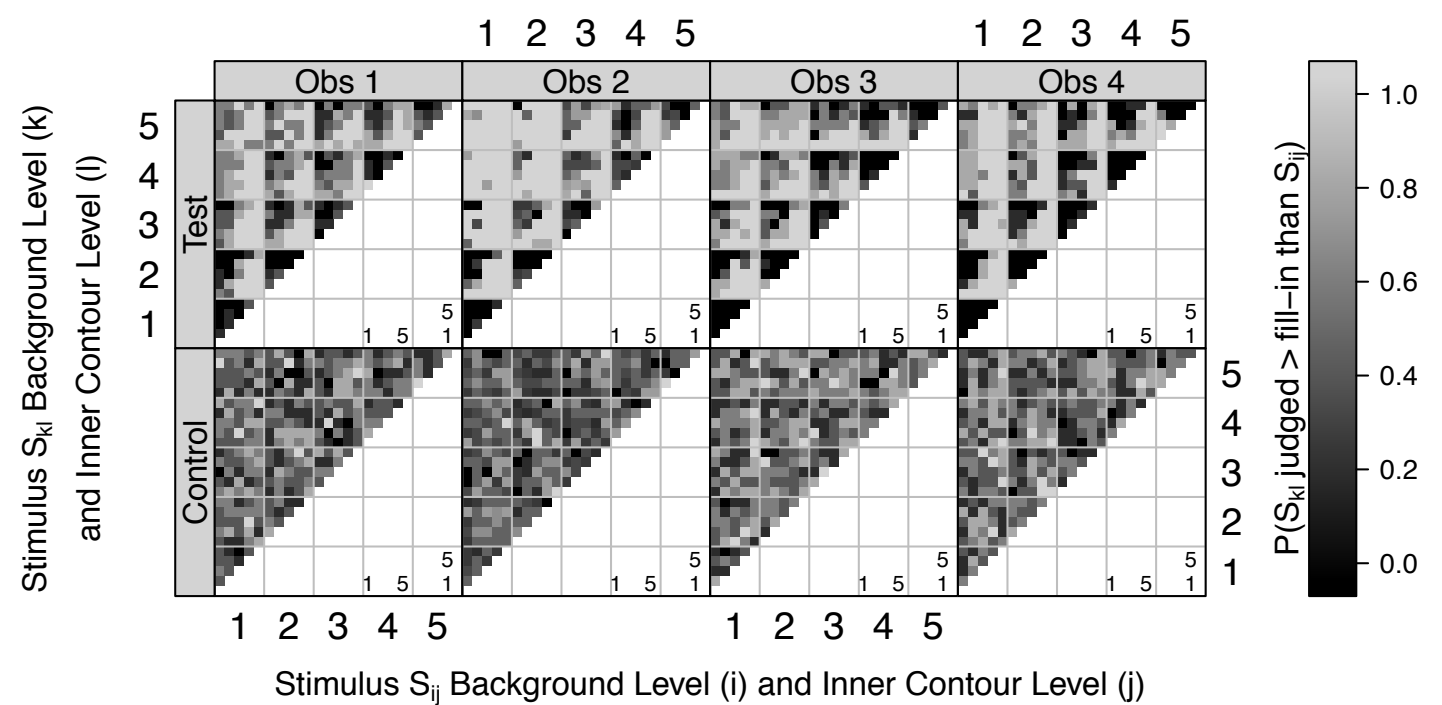

(c)

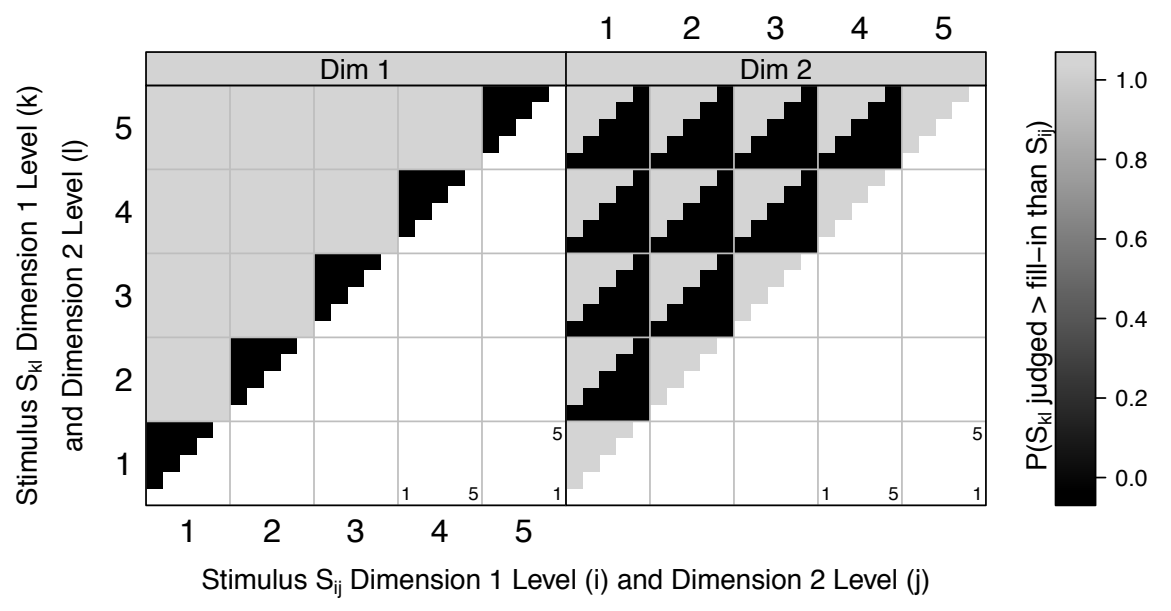

Figure 3 
(a)

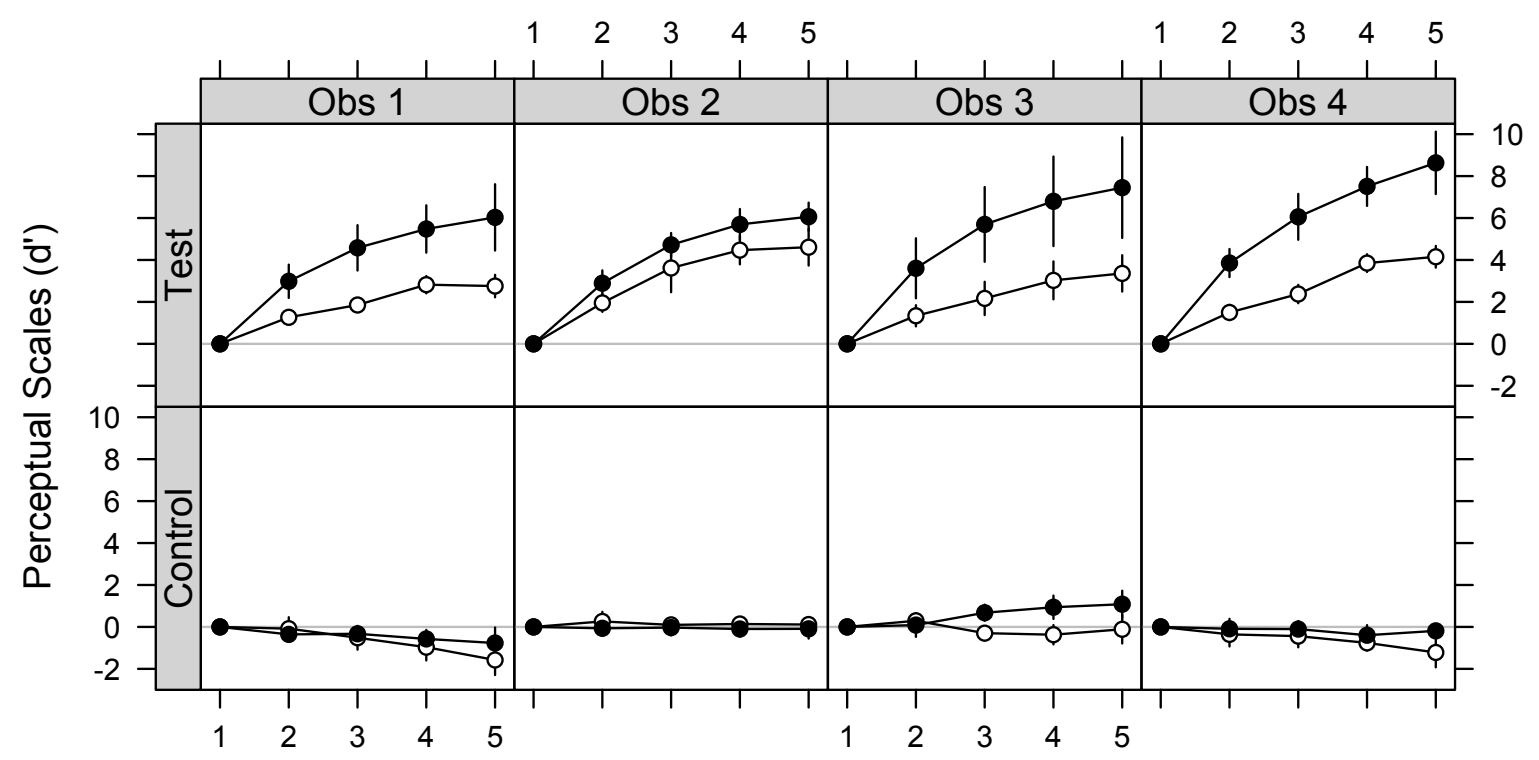

Physical Scale Index

(b)

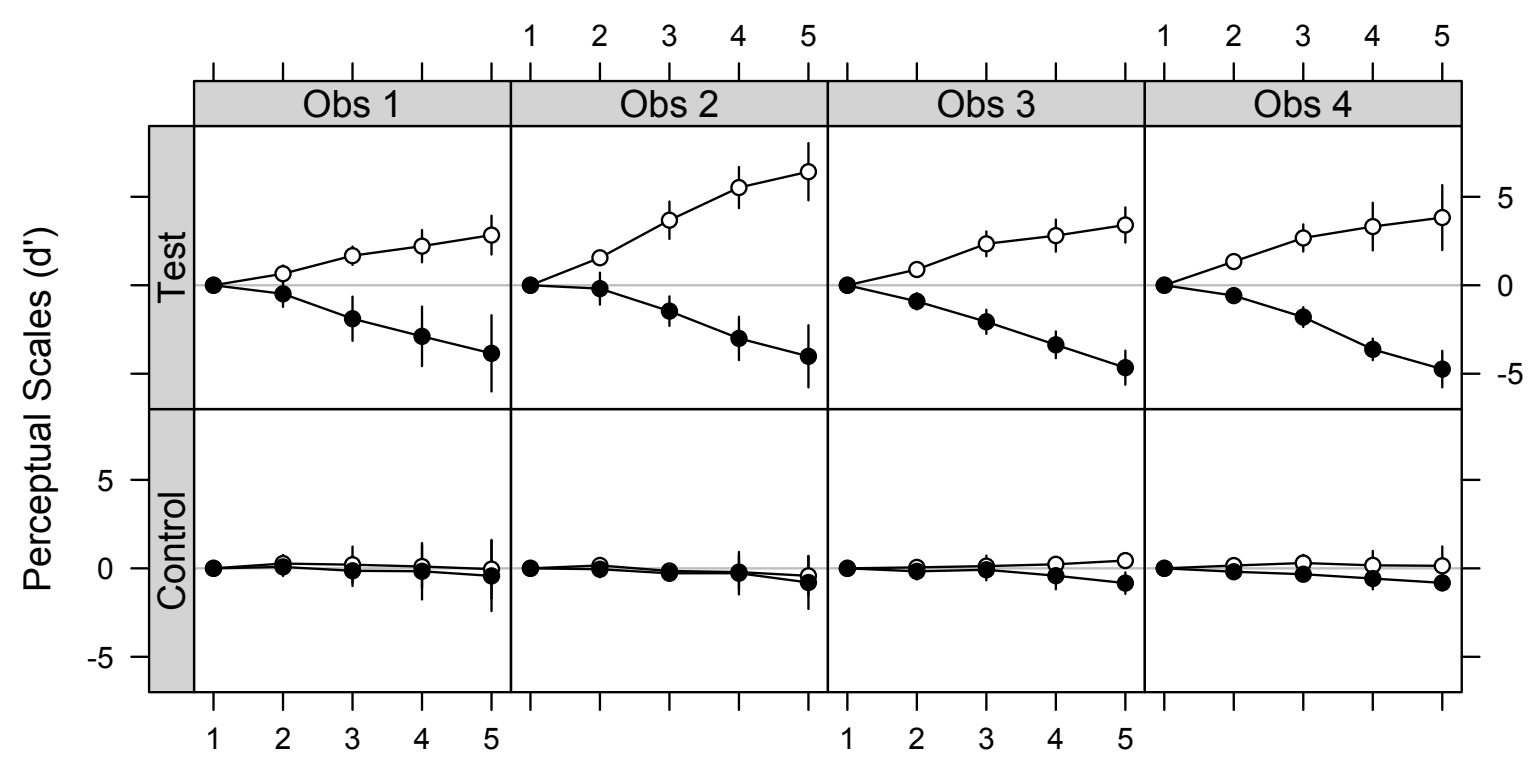

Physical Scale Index

Figure 4 
(a)

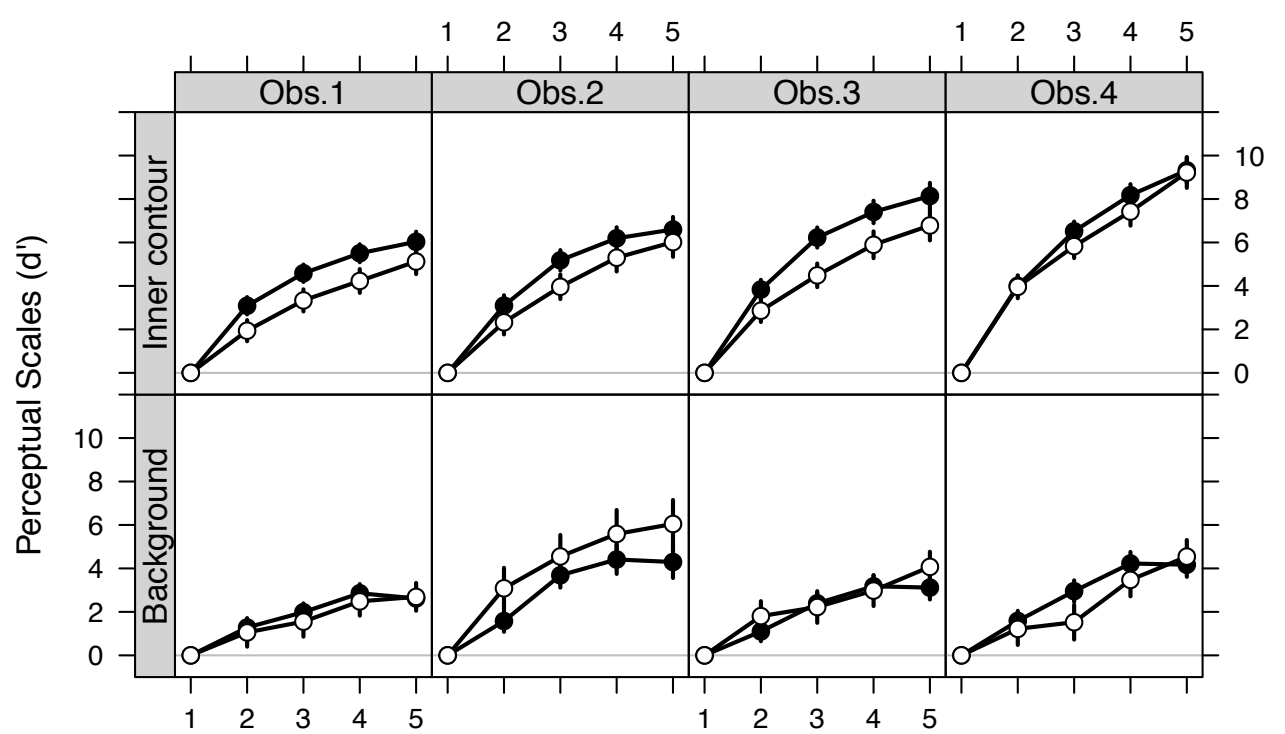

Physical Scale Index

(b)

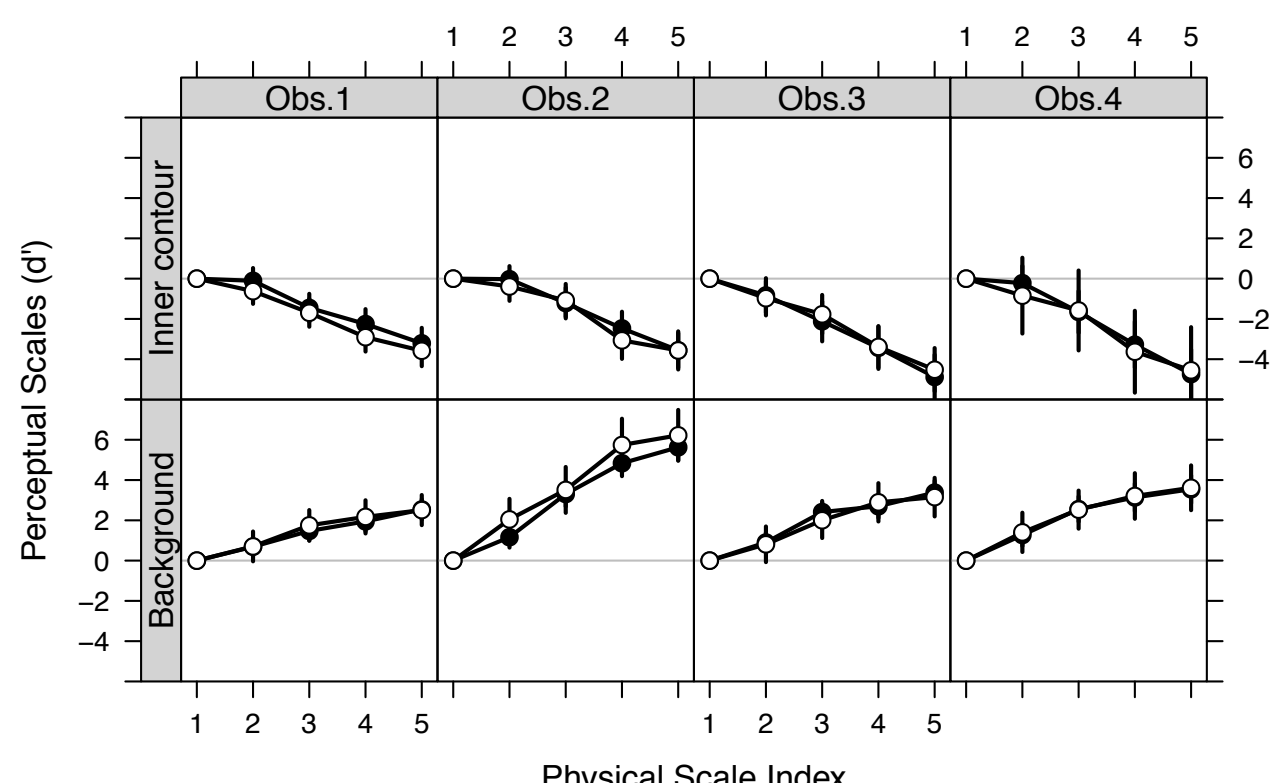

Physical Scale Index

\section{Order}

$\mathrm{B} 1$ then $\mathrm{B} 2$

B2 then B1

\section{Order}

B1 then B2
B2 then B1

Figure 5 
(a)

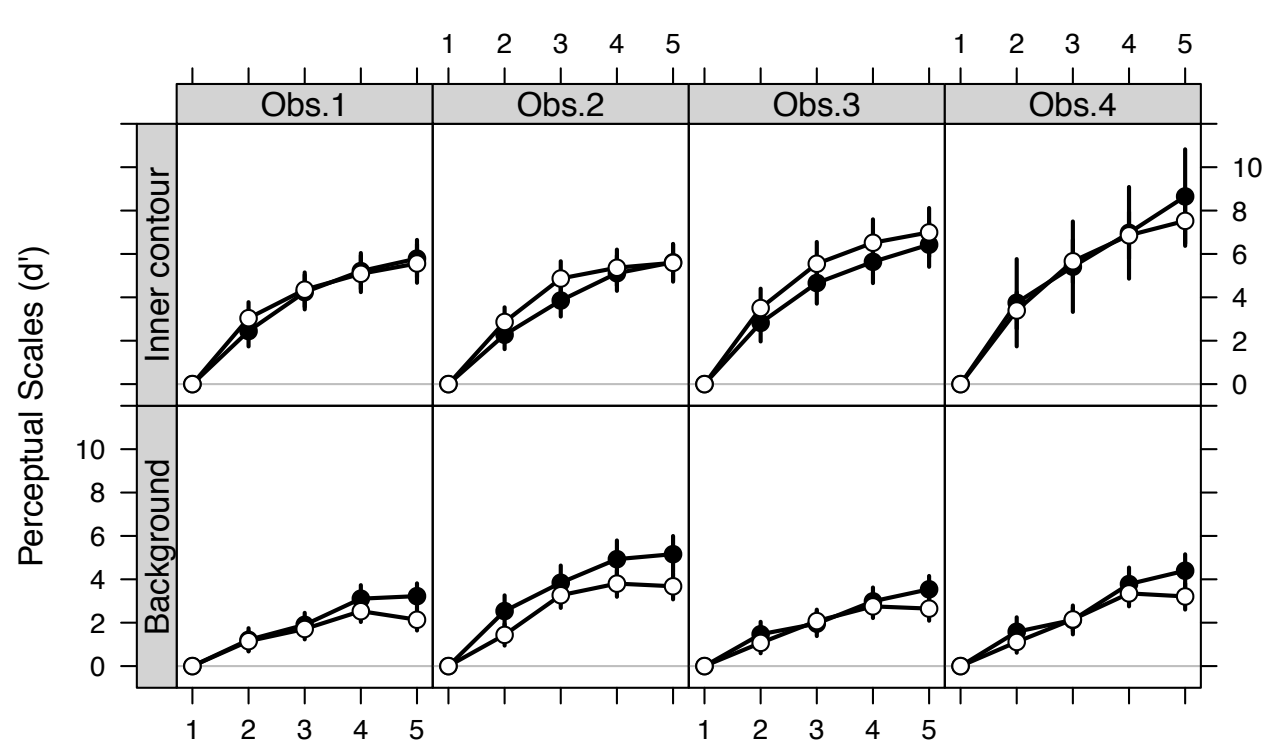

Physical Scale Index

\section{Order}

$\mathrm{C} 1$ then $\mathrm{C} 2$

$\mathrm{C} 2$ then $\mathrm{C} 1$

(b)

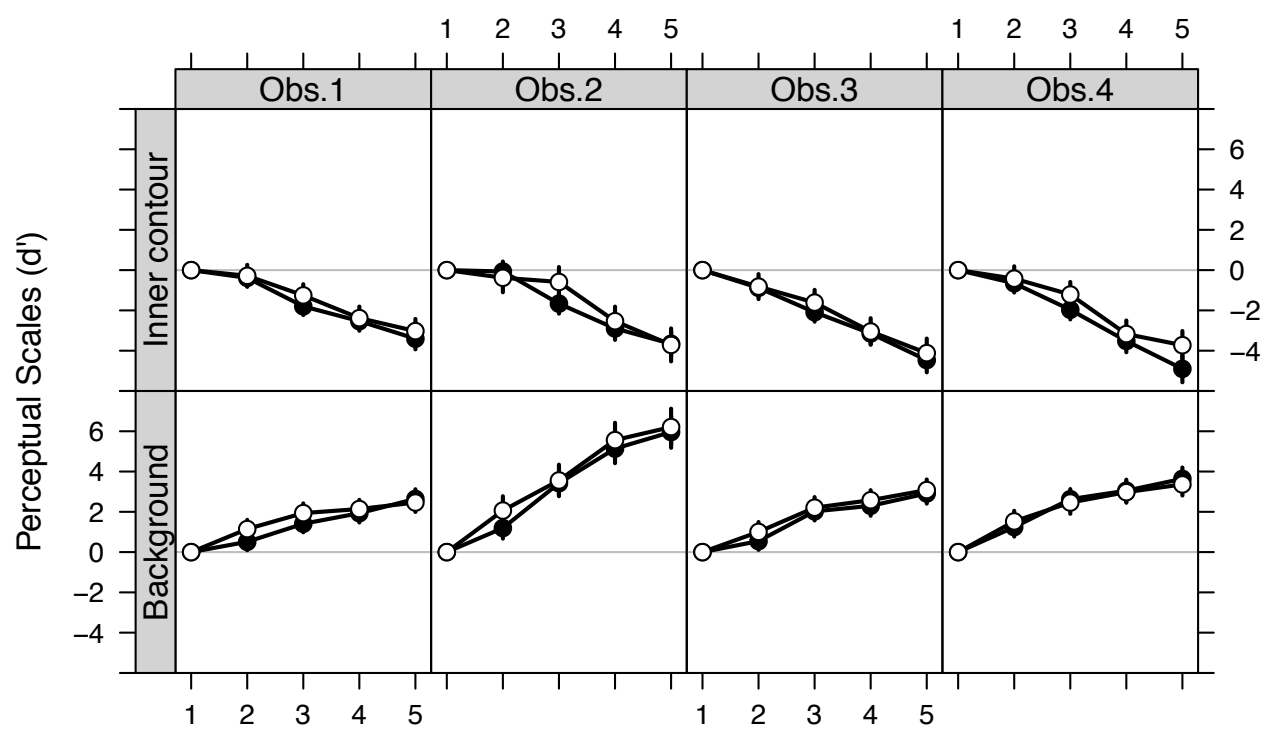

Physical Scale Index
Order

C1 then $\mathrm{C} 2$

$\mathrm{C} 2$ then $\mathrm{C} 1$

Figure 6 

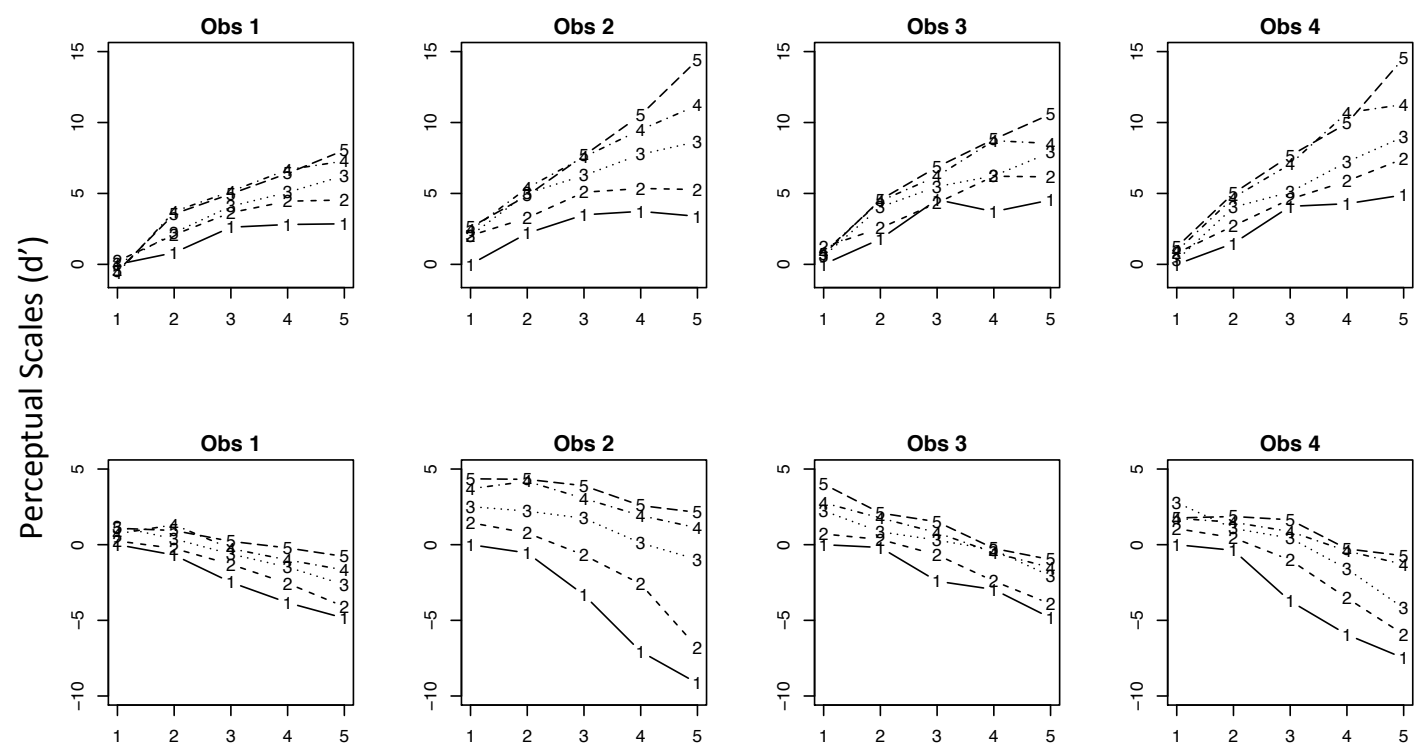

Physical Scale Index (Interior Contour Luminance)

Figure 7 
a

Hue

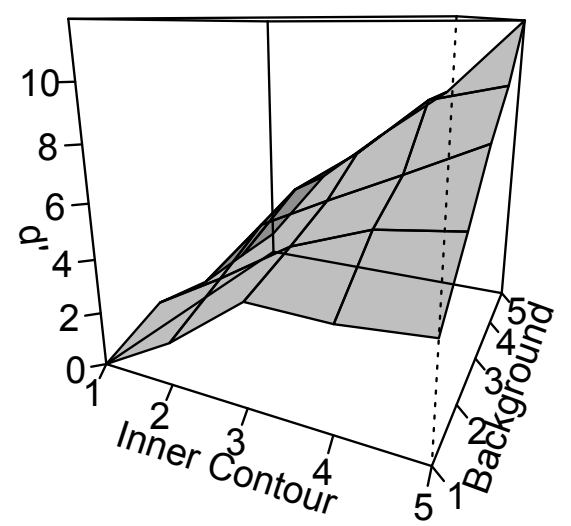

b

Brightness

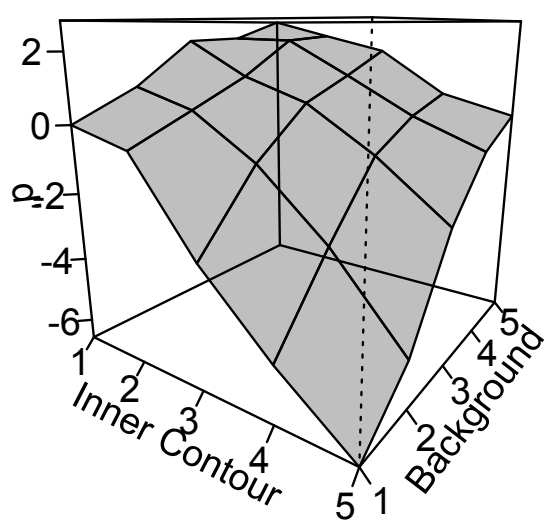

Figure 8 
Table I: Comparison of independence and additive models for judgements based on hue

\begin{tabular}{cccc}
\hline & \multicolumn{3}{c}{ Test stimuli (additive model $\times$ independent } \\
& model) & p-value \\
\cline { 2 - 4 } & Df & Deviance & $<0.001$ \\
Obs. 1 & 4 & 270.22 & $<0.001$ \\
Obs. 2 & 4 & 568.41 & $<0.001$ \\
Obs. 3 & 4 & 309.60 & $<0.001$ \\
Obs. 4 & 4 & 406.38 & \\
\hline
\end{tabular}

Table II: Comparison of independence and additive models for judgement based on brightness

\begin{tabular}{cccc}
\hline & \multicolumn{3}{c}{ Test stimuli (additive model $\times$ independent } \\
& model) & \\
\cline { 2 - 4 } & Df & Deviance & p-value \\
\hline Obs. 1 & 4 & 275.434 & $<0.001$ \\
Obs. 2 & 4 & 912.11 & $<0.001$ \\
Obs. 3 & 4 & 400.82 & $<0.001$ \\
Obs. 4 & 4 & 430.64 & $<0.001$ \\
\hline
\end{tabular}


Table III: Comparison of additive and saturated models for judgement based on hue

\begin{tabular}{cccc}
\hline & \multicolumn{3}{c}{ Test stimuli (additive model $\times$ saturated } \\
& model) & p-value \\
\cline { 2 - 4 } & Df & Deviance & $<0.001$ \\
Obs. 1 & 16 & 105.44 & $<0.001$ \\
Obs. 2 & 16 & 142.82 & $<0.001$ \\
Obs. 3 & 16 & 104.81 & $<0.001$ \\
Obs. 4 & 16 & 108.14 & \\
\hline
\end{tabular}

Table IV: Comparison of additive and saturated models for judgement based on brightness

\begin{tabular}{cccc}
\hline & \multicolumn{3}{c}{ Test stimuli (additive model $\times$ saturated } \\
& model) & \\
\cline { 2 - 4 } & Df & Deviance & p-value \\
\hline Obs. 1 & 16 & 56.154 & $<0.001$ \\
Obs. 2 & 16 & 74.01 & $<0.001$ \\
Obs. 3 & 16 & 32.83 & $<0.01$ \\
Obs. 4 & 16 & 122.78 & $<0.001$ \\
\hline
\end{tabular}

